\section{International Scientific Journal Theoretical \& Applied Science}

\author{
p-ISSN: 2308-4944 (print) e-ISSN: 2409-0085 (online) \\ Year: 2015 Issue: 10 Volume: 30
}

Published: $30.10 .2015 \quad$ http://T-Science.org

SECTION 8. Architecture and construction.
Andrei Mastislavovich Korneev

Doctor of Technical Sciences, Professor, director of the institute of Lipetsk State Technical University, Russia, Lipetsk

Olga Petrovna Buzina

Candidate of Technical Sciences, Associate Professor, head of the department of Lipetsk State Technical University, Russia, Lipetsk

Andrei Vladimirovich Sukhanov postgraduate student, assistant of Lipetsk State Technical University, Russia, Lipetsk

Ilya Andreevich Shipulin postgraduate student, assistant of Lipetsk State Technical University, Russia, Lipetsk shipulin-ilya@yandex.ru

Nikita Andreevich Shipulin student of Lipetsk State Technical University, Russia, Lipetsk

\title{
THE STUDY OF THE MECHANICAL PROPERTIES OF FINE-GRAINED CINDER CONCRETE UNDER AXIAL TENSION AND COMPRESSION
}

Abstract: The article considers the results of study the mechanical properties of fine-grained cinder concrete under axial tension and compression. The dependences that are connected with the cube strength of the concrete such characteristics of fine-grained concrete as the tensile strength, tangent modulus of elasticity, limiting relative deformation under tension and compression have been identified, according to the results of experiments.

Key words: mechanical properties of concrete, fine-grained concrete, axial tension and compression, testing experimental samples.

Language: Russian

Citation: Korneev AM, Buzina OP, Sukhanov AV, Shipulin IA, Shipulin NA (2015) THE STUDY OF THE MECHANICAL PROPERTIES OF FINE-GRAINED CINDER CONCRETE UNDER AXIAL TENSION AND COMPRESSION. ISJ Theoretical \& Applied Science 10 (30): 65-71.

Soi: http://s-o-i.org/1.1/TAS-10-30-16 Doi: crossef http://dx.doi.org/10.15863/TAS.2015.10.30.16

\section{ИССЛЕДОВАНИЕ МЕХАНИЧЕСКИХ СВОЙСТВ МЕЛКОЗЕРНИСТОГО ШЛАКОБЕТОНА ПРИ ОСЕВОМ РАСТЯЖЕНИИ И СЖАТИИ}

Аннотация: В статье приводятся результаты исследования механических свойств мелкозернистого шлакобетона. По результатам опытов выявлены зависимости, связывающие такие характеристики мелкозернистого шлакобетона, как прочность при растяжении, начальный модуль упругости, предельные относительные деформации при растяжении и сжатии с кубиковой прочностью бетона.

Ключевые слова: механические свойства бетона, мелкозернистый илакобетон, осевое растяжение и сжатие, испытания опытных образиов.

В связи с возрастающими требованиями к эффективному и экономичному потреблению материальных и энергетических ресурсов, а также к снижению стоимости и трудоёмкости технологических процессов, в настоящее время происходит интенсивный рост области применения железобетонных конструкций из бетонов, позволяющих использовать для их производства вторичные отходы различных отраслей промышленности и местные заполнители. К таким бетонам, в частности, относится мелкозернистый шлакобетон на основе отсева от дробления литого шлакового щебня.

Несмотря на столь широкое применение шлакобетонных и сталефиброшлакобетонных конструкций в современном строительстве, многие особенности их прочностных характеристик мало изучены. В частности, это относится к проблеме определения физикомеханических характеристик шлакобетона, 
подбора для него зависимостей и параметров для расчёта элементов конструкций по диаграммной методике [1].

Целью настоящего исследования является изучение физико-механических характеристик мелкозернистого шлакобетона, получение зависимостей и параметров, необходимых для достоверного расчёта элементов шлакобетонных конструкций по диаграммной методике.

Значительная часть расчётов элементов строительных конструкций основывается на таких характеристиках материала, как призменная прочность бетона $R_{b}$, кубиковая прочность $R_{m}$, прочность при осевом растяжении $R_{b t}$, начальный модуль упругости $E_{b}^{0}$ бетона, а также предельные относительные деформации бетона при сжатии $\varepsilon_{b R}$ и растяжении $\varepsilon_{b t R}$.
Основным источником информации о механических свойствах материала, содержащий данные величины, являются диаграммы деформирования бетона, связывающие относительные деформации с напряжениями «є б» при одноосном сжатии и растяжении, для получения которых проводились испытания на образцах, изготовленных на основе шлаков металлургического производства ОАО «НЛМК». В исследуемых составах использовались в качестве вяжущего цемент марки ПЦ-500 D0 Липецкого цементного завода и пластифицирующая добавка Реламикс (10\% раствор), а в качестве заполнителя - отсев от дробления литого шлакового щебня фракции $0 . .5$ мм с насыпной плотностью от 1085 до 1135 кг $/ \mathrm{m}^{3}$.

Составы экспериментальных образцов представлены в таблице 1 .

Таблица 1

Составы бетона для каждой партии образцов.

\begin{tabular}{|c|c|c|c|c|}
\hline \multirow[b]{2}{*}{ № состава } & \multirow[b]{2}{*}{ Класс (марка) } & \multicolumn{3}{|c|}{ Расход компонентов, кг/м ${ }^{3}$} \\
\hline & & Цемент & $\begin{array}{c}\text { Отсев от дробления литого } \\
\text { шлакового щебня }\end{array}$ & Вода \\
\hline 1 & B3,5 (M50) & 330 & 1498 & 205 \\
\hline 2 & B5 (M75) & 335 & 1520 & 200 \\
\hline 3 & B7,5 (M100) & 350 & 1555 & 190 \\
\hline 4 & $\mathrm{~B} 10(\mathrm{M} 150)$ & 365 & 1510 & 180 \\
\hline 5 & B12,5 (M150) & 400 & 1560 & 185 \\
\hline 6 & B15 (M200) & 420 & 1510 & 190 \\
\hline 7 & $\mathrm{~B} 20 \mathrm{M}(250)$ & 440 & 1463 & 200 \\
\hline
\end{tabular}

Твердение бетона происходило в лабораторных условиях при температуре +18-20 ${ }^{\circ} \mathrm{C}$ и влажности $70 \pm 5 \%$. Нагружение шлакобетона при испытании на сжатие происходило со скоростью $0,6 \pm 0,4 \mathrm{MПа} / \mathrm{c}$, при испытании на растяжение $-0,05 \pm 0,02 \mathrm{MПа} / \mathrm{c}$.

Прочность бетона на сжатие и растяжение определялась на стандартных образцах согласно указаниям ГОСТ 10180-90. Кубиковая прочность определялась на образцах 7х7х7 см, призменная прочность и прочность на растяжение определялись на призмах 7х7×28 см. Относительные деформации при испытании призм на растяжение и сжатие определялись с помощью индикаторов часового типа с ценой деления 0,001 мм на базе 180 мм. Численные результаты испытаний представлены в таблице 2 , эмпирические диаграммы «в - б» представлены на рисунке $1 \mathrm{a}$, б.

\section{Результаты испытания опытных образцов на осевое растяжение и сжатие}

Таблица 2

\begin{tabular}{|c|c|c|c|c|c|c|}
\hline \multirow{3}{*}{$\begin{array}{c}\text { № } \\
\text { состава }\end{array}$} & \multirow{3}{*}{$\begin{array}{c}\text { Средние } \\
\text { значения } \\
\text { начального } \\
\text { модуля } \\
\text { упругости } E_{b}^{0} \text {, } \\
\text { МПа }\end{array}$} & \multicolumn{3}{|c|}{ Средние значения прочности бетона, МПа } & \multicolumn{2}{|c|}{$\begin{array}{c}\text { Средние значения } \\
\text { относительных деформаций } \\
\text { в вершине диаграммы }\end{array}$} \\
\hline & & \multicolumn{2}{|c|}{ при сжатии } & \multirow[b]{2}{*}{$\begin{array}{c}\text { при осевом } \\
\text { растяжении } \\
R_{b t}\end{array}$} & \multirow{2}{*}{$\begin{array}{c}\text { при } \\
\text { сжатии } \\
\varepsilon_{b R} \cdot 10^{-3}\end{array}$} & \multirow{2}{*}{$\begin{array}{c}\text { при осевом } \\
\text { растяжении } \\
\varepsilon_{b t R} \cdot 10^{-3}\end{array}$} \\
\hline & & $\begin{array}{c}\text { кубиковая } \\
\text { прочность, } \\
R_{m}\end{array}$ & $\begin{array}{c}\text { призменная } \\
\text { прочность } R_{b}\end{array}$ & & & \\
\hline 1 & 8,036 & 4,58 & 4,44 & 1,115 & 1,397 & 0,154 \\
\hline 2 & 9,453 & 7,06 & 6,71 & 1,362 & 1,603 & 0,169 \\
\hline 3 & 11,594 & 8,78 & 8,60 & 1,679 & 1,741 & 0,178 \\
\hline 4 & 15,248 & 15,15 & 15,10 & 2,099 & 2,101 & 0,202 \\
\hline 5 & 17,663 & 19,06 & 18,30 & 3,079 & 2,239 & 0,211 \\
\hline
\end{tabular}

ISPC Innovations in science, 


\begin{tabular}{|c|c|c|c|c|c|}
\hline Impact Factor: & $\begin{array}{l}\text { ISRA (India) } \\
\text { ISI (Dubai, UAI } \\
\text { GIF (Australia) } \\
\text { JIF }\end{array}$ & $\begin{array}{l}=1.344 \\
=0.829 \\
=0.356 \\
=1.500\end{array}$ & $\begin{array}{l}\text { SIS (USA) } \\
\text { PИНЦ (Russia) } \\
\text { ESJI (KZ) } \\
\text { SJIF (Morocco) }\end{array}$ & $\begin{array}{l}=0.912 \\
=0.179 \\
=1.042 \\
=2.031\end{array}$ & ICV (Poland) \\
\hline
\end{tabular}

\begin{tabular}{|l|l|l|l|l|l|l|}
\hline 6 & 19,715 & 22,69 & 22,01 & 3,154 & 2,382 & 0,219 \\
\hline 7 & 21,606 & 28,81 & 27,60 & 3,415 & 2,568 & 0,231 \\
\hline
\end{tabular}

a)

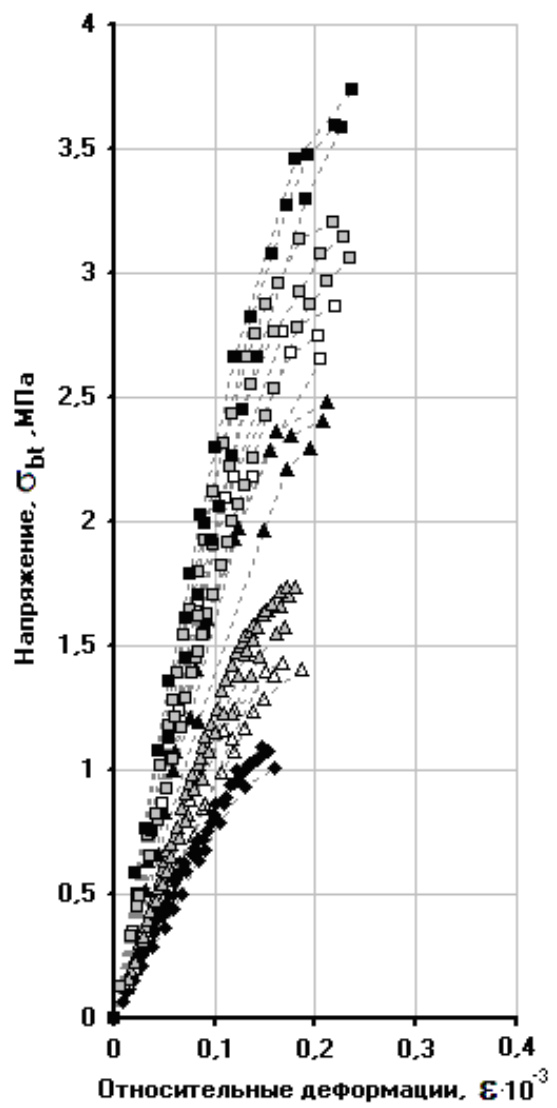

6]

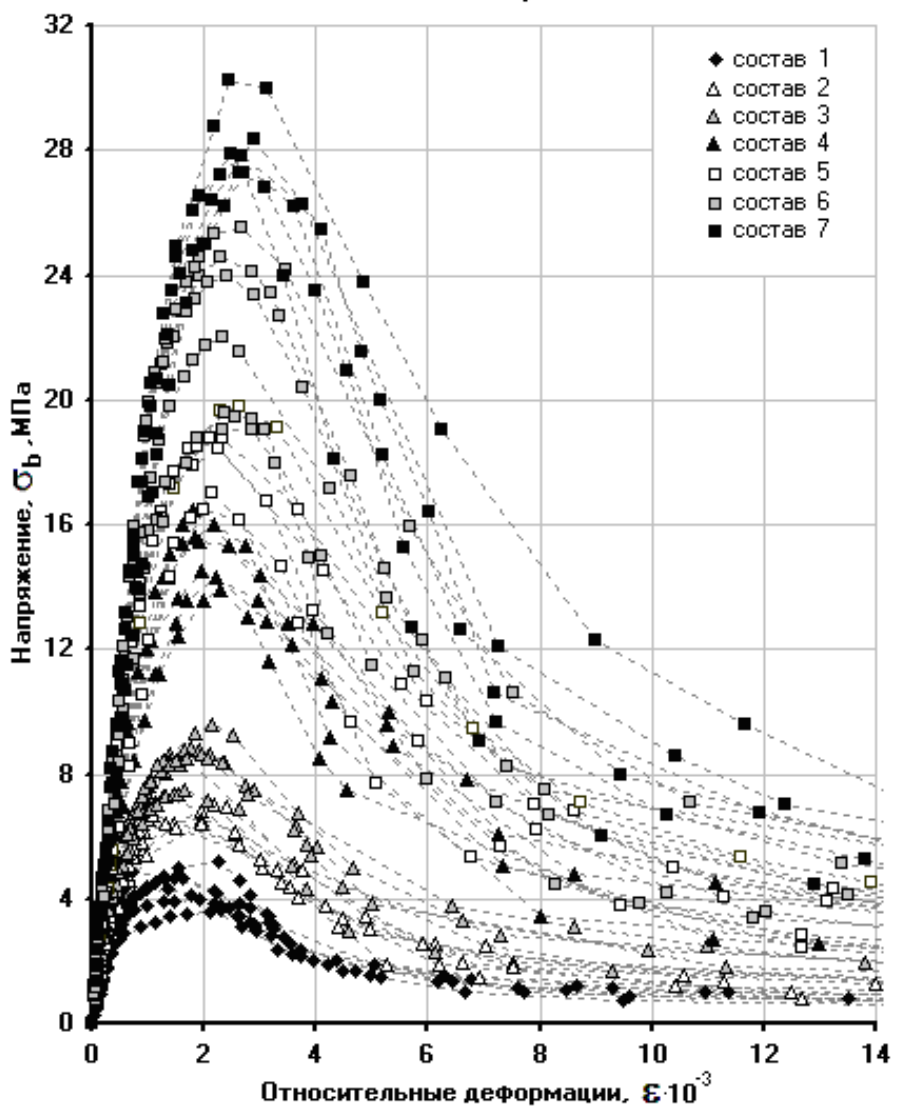

Рисунок 1 - Эмпирические диаграммы «є - б» для мелкозернистого шлакобетона: а - при осевом растяжении, б - при сжатии.

При проектировании оптимальных составов сталефибробетонных смесей, расчёте риска возникновения трещин в конструкциях из сталефибробетона, а также для разработки автоматизированных программ, ведущих расчёт конструкций по диаграммной методике используются такие физико-механические характеристики бетона, как кубиковая прочность $R_{m}$, прочность на растяжение $R_{b t}$, начальный модуль упругости $E_{b}^{0}$, значения предельных относительных деформаций бетона при растяжении $\varepsilon_{b t R}$ и сжатии $\varepsilon_{b R}$. На практике удобно пользоваться величиной $R_{m}$, в связи с чем многие специалисты проводят исследования по поиску зависимостей, выражающих характеристики отдельного вида бетона через его кубиковую прочность.

Общеизвестна формула, связывающая предел прочности бетона при растяжении $R_{b t}$ с его кубиковой прочностью $R_{m}$ :

$$
R_{b t}=0,5 \sqrt[3]{R_{m}^{2}}
$$

В работе [2] предложена следующая зависимость для мелкозернистого песчаного бетона:

$$
R_{b t}=0,3 \sqrt[3]{R_{m}^{2}}
$$

Из наших опытов установлено, что прочность при осевом растяжении образцов из мелкозернистого шлакобетона составляет приблизительно 0,08...0,3 прочности при сжатии. В результате обработки опытных данных удалось получить следующую зависимость:

$$
R_{b t}=0,4 \sqrt[3]{R_{m}^{2}}
$$

Опытные и полученные по формулам (1)-(3) зависимости прочности при растяжении от прочности при сжатии приведены на рисунке 2 . 


\begin{tabular}{|c|c|c|c|c|c|c|}
\hline Impact Factor: & $\begin{array}{l}\text { ISRA (India) } \\
\text { ISI (Dubai, UAF } \\
\text { GIF (Australia) } \\
\text { JIF }\end{array}$ & $\begin{array}{l}=1.344 \\
=0.829 \\
=0.356 \\
=1.500\end{array}$ & $\begin{array}{l}\text { SIS (USA) } \\
\text { PИНЦ (Russia) } \\
\text { ESJI (KZ) } \\
\text { SJIF (Morocco) }\end{array}$ & $\begin{array}{l}=0.912 \\
=0.179 \\
=1.042 \\
=2.031\end{array}$ & ICV (Poland) & $=6.630$ \\
\hline
\end{tabular}

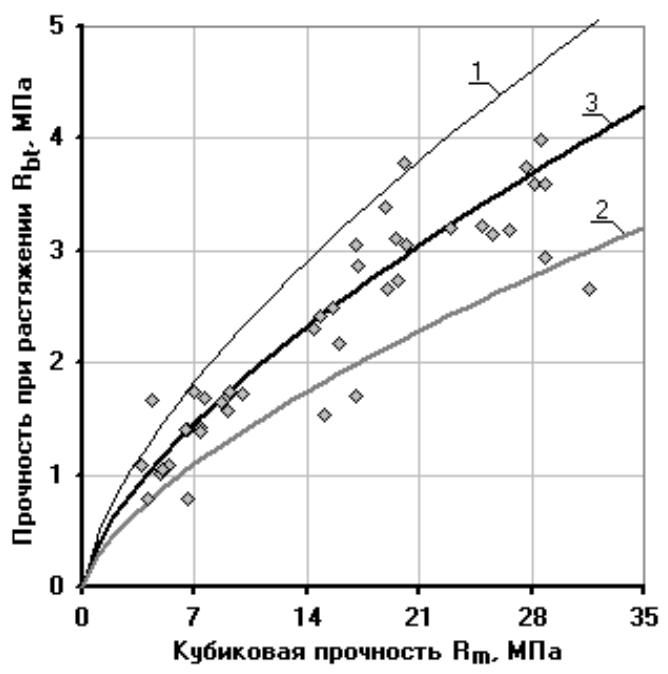

Рисунок 2 - Зависимость прочности при растяжении $R_{b t}$ от кубиковой прочности $R_{m}: 1$ - по формуле (1), 2 - по формуле (2), 3 - по формуле (3).

Начальный модуль упругости бетона $E_{b}^{0}$ удобно вычислять через $R_{m}$ и объёмный вес бетона (плотность) $\rho$, кг $/ \mathrm{M}^{3}$.

С целью определения зависимости объёмной массы мелкозернистого шлакобетона $\rho$ от его кубиковой прочности $R_{m}$ были проведены отдельные испытания на образцах бетона размером $7 \times 7 \times 7$ см. Численные значения экспериментальных данных представлены в таблице 3.

Таблица 3

Опытные значения кубиковой прочности и объёмной массы шлакобетонных образцов.

\begin{tabular}{|c|c|c|}
\hline Класс (марка) & Кубиковая прочность, $R_{m}$, МПа & Объёмная масса, $\rho$, кг/м ${ }^{3}$ \\
\hline \multirow{3}{*}{ B7,5 (M100) } & 11,98 & 2014,4 \\
\hline & 12,12 & 2039,1 \\
\hline & 11,62 & 2025,7 \\
\hline \multirow{3}{*}{ B10 (M150) } & 14,21 & 2026,9 \\
\hline & 14,20 & 2032,9 \\
\hline & 13,70 & 2052,5 \\
\hline \multirow{3}{*}{ B12,5 (M150) } & 17,49 & 2086,9 \\
\hline & 17,27 & 2060,8 \\
\hline & 16,42 & 2072,8 \\
\hline \multirow{3}{*}{ B20 (M250) } & 32,40 & 2131,1 \\
\hline & 31,30 & 2133,2 \\
\hline & 29,81 & 2113,9 \\
\hline \multirow{3}{*}{ B22,5 (M300) } & 34,45 & 2111,6 \\
\hline & 34,16 & 2148,8 \\
\hline & 33,90 & 2125,0 \\
\hline
\end{tabular}

Анализ данных, представленных в таблице 2, позволил получить следующую зависимость для выражения объёмной массы мелкозернистого шлакобетона от его кубиковой прочности:

$$
\rho=100 \cdot \ln \left(R_{m}\right)+1780
$$

где $R_{m}$ - кубиковая прочность бетона в МПа; $\rho$ -

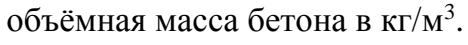

Для выражения начального модуля упругости $E_{b}^{0}$ (в МПа) применительно к мелкозернистому шлакобетону получена формула:

$$
\begin{aligned}
& E_{b}^{0}=1,95 \rho \sqrt{R_{m}}= \\
& =1,95 \cdot\left(100 \cdot \ln \left(R_{m}\right)+1780\right) \sqrt{R_{m}}
\end{aligned}
$$




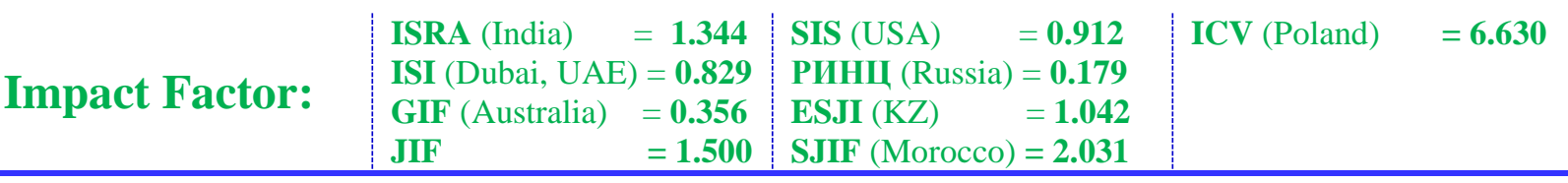

Соотношение опытных данных с теоретической кривой, построенной по формуле (5) позволяет сделать вывод о применимости

данной зависимости для выражения начального модуля упругости мелкозернистого шлакобетона через его кубиковую прочность (рис. 3).

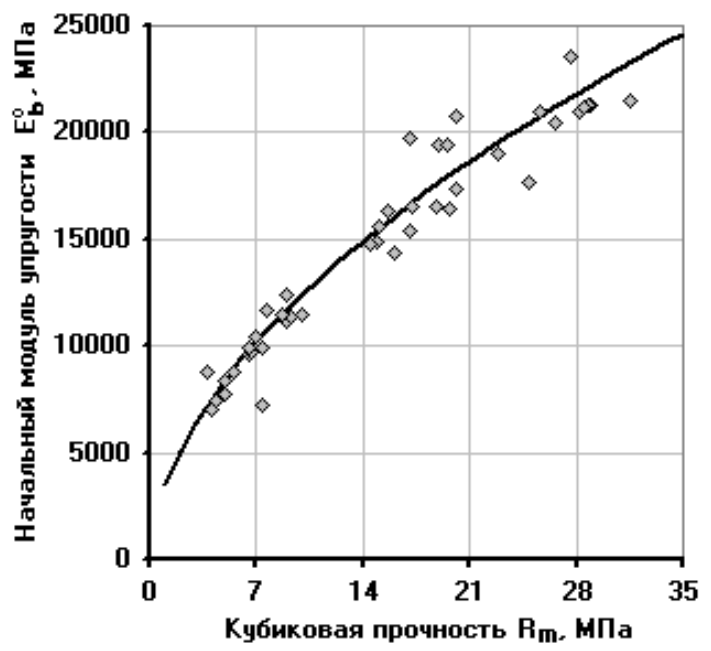

Рисунок 3 - Опытные данные и теоретическая кривая зависимости начального модуля упругости мелкозернистого шлакобетона от его кубиковой прочности.

Получены зависимости для определения предельных относительных деформаций мелкозернистого шлакобетона:

$$
\varepsilon_{b R}=0,85 \cdot \sqrt[3]{R_{m}} \cdot 10^{-3},
$$

для сжатия и

$$
\varepsilon_{b t R}=0,15 \cdot \sqrt[3]{R_{b t}} \cdot 10^{-3},
$$

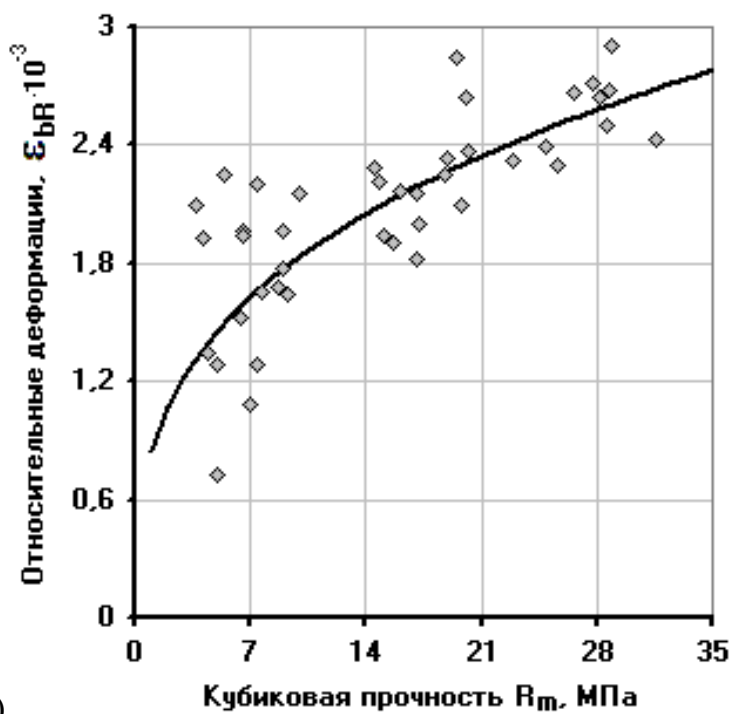

a)

Кубиковая прочность $R_{\mathrm{m}}$. МПа для растяжения. Подставляя (3) в (7) получаем следующую формулу для выражения $\varepsilon_{b t R}$ через кубиковую прочность:

$$
\varepsilon_{b t R}=0,11 \cdot \sqrt[9]{R_{m}^{2}} \cdot 10^{-3},
$$

Соотношение опытных данных с теоретическими кривыми для $\varepsilon_{b t R}$ и $\varepsilon_{b R}$ показано на рисунке 4.

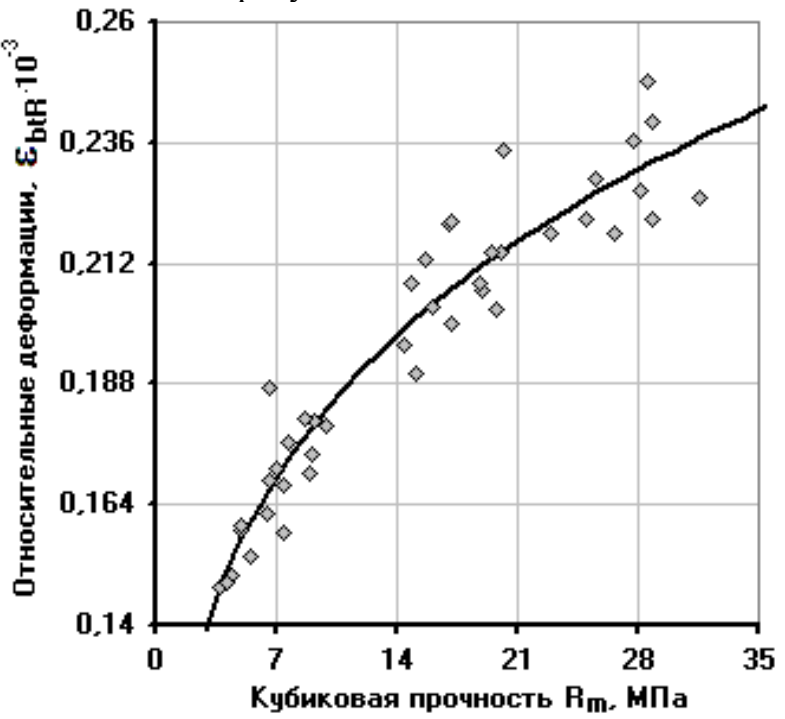

Рисунок 4 - Зависимость относительных деформаций в вершине диаграммы «є - б» от кубиковой прочности $R_{m}$ : a - при сжатии, кривая построена по формуле (6); б - при осевом растяжении, кривая построена по формуле (8).

В настоящее время имеется немало

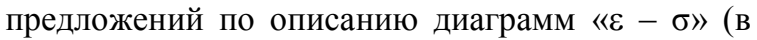
виде полинома, степенной функции, показательной функции и других зависимостей). Та или иная зависимость может быть выбрана с учётом особенностей решаемой задачи $[3,4,5,6]$.

ISPC Innovations in science, 


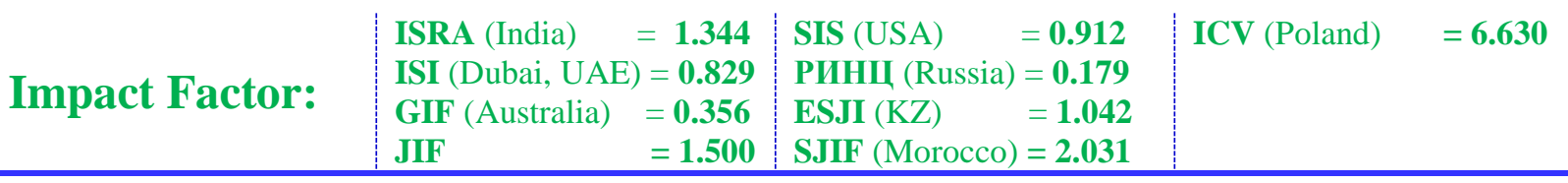

В данном случае оказалось удобным использовать зависимость ЕКБ-ФИП:

$$
\frac{\sigma_{b}}{R_{b}}=\frac{\left(\frac{E_{b}^{0} \varepsilon_{b R}}{R_{b}}\right)\left(\frac{\varepsilon_{b}}{\varepsilon_{b R}}\right)-\left(\frac{\varepsilon_{b}}{\varepsilon_{b R}}\right)^{2}}{1+\left(\frac{E_{b}^{0} \varepsilon_{b R}}{R_{b}}-2\right) \frac{\varepsilon_{b}}{\varepsilon_{b R}}},
$$

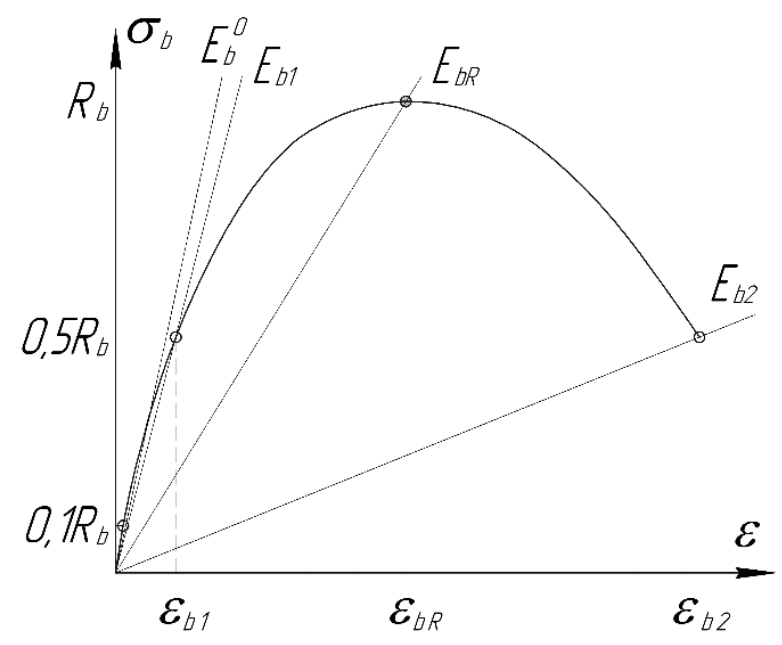

Рисунок 5 - Диаграмма « $\varepsilon$ - б» согласно ЕКБ-ФИП.
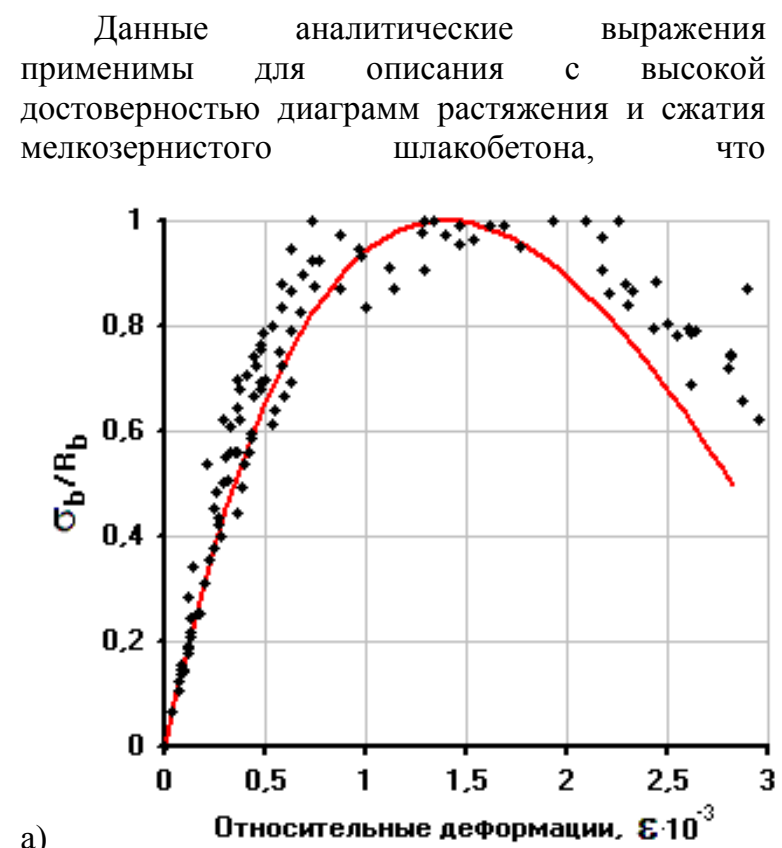

a)
Кривая зависимости (9) представлена на рисунке 5. При построении диаграммы растяжения индекс «b» в этом выражении заменяется на «bt». 
напряжения ниспадающую ветвь использовать в расчётах не рекомендуется[7].

Выводы. Из опытов установлено, что прочность мелкозернистого шлакобетона при осевом растяжении составляет приблизительно 0,08...0,3 прочности при сжатии. На основе экспериментальных данных получены зависимости, выражающие такие характеристики мелкозернистого шлакобетона, как прочность при растяжении, начальный модуль упругости, предельные относительные деформации при растяжении и сжатии, через кубиковую прочность.

Для описание диаграмм деформирования мелкозернистого шлакобетона принята зависимость, рекомендуемая ЕКБ-ФИП.

\section{References:}

1. Chernousov RN (2011) Strength and deformation of structural elements on the basis of transport facilities fine steel fiber cinder concrete. Nauchnyi vestnik Voronezhskogo gosudarstvennogo arkhitekturno-stroitel'nogo universiteta. Stroitel'stvo i arkhitektura. 2011. No 1(21), pp. 87-97. (In Russian).

2. Chernousov NN, Chernousov RN, Sukhanov AV (2013) Modeling of strength and deformation properties of fine-grained sandcement concrete under axial tension and compression. Stroitel'nye Materialy. 2013. No 10, pp. 12-14. (In Russian).

3. (2002) Atlas of stress-strain curves. Second edition. Materials Park, USA, 2002, 816 p.

4. Murashkin GV, Mordovskii SS (2013) Application strain diagrams for calculating the bearing capacity of eccentrically compressed concrete elements. Zhilishchnoe stroitel'stvo. 2013. No 3, pp. 38-40. (In Russian).
5. Murashkin G, Panfilov D, Murashkin V (2013) An improved technique of calculating deflections of flexural reinforced concrete elements made of conventional and highstrenght concrete // Journal of Civil Engineering and Architecture. USA. 2013. Vol. 7, No. 2 (Serial number 63), pp. 125-131.

6. Panfilov DA, Pishulev AA, Gimadetdinov KI (2014) Review of existing strain diagrams of concrete in compression in domestic and foreign regulations. Promyshlennoe i grazhdanskoe stroitel'stvo. 2014. No 3. pp. 8084. (In Russian).

7. Chernousov NN, Chernousov RN, Sukhanov AV (2014) Research mechanic work of finegrained cinder concrete under axial tension and compression. Stroitel'nye Materialy. 2014. No 12, pp. 59-63. (In Russian). 